\title{
Deterioro del flujo microvascular coronario durante la angioplastía primaria en pacientes consumidores de cocaína
}

\author{
Nicolás Veas ${ }^{2}$, Dante Lindefjeld ${ }^{1-2}$, José Luis Winter ${ }^{2}$, Manuel Méndez ${ }^{1-2}$, Osvaldo Pé- \\ rez $^{1-2}$, Alejandro Martínez ${ }^{2}$, Eduardo Guarda ${ }^{2}$
}

\author{
1 Laboratorio de Hemodinamia Hospital Sótero del Rio. \\ 2 Departamento Enfermedades Cardiovasculares, \\ Pontificia Universidad Católica de Chile, Santiago.
}

Introducción y objetivo: El consumo de cocaína puede inducir deterioro del flujo microvascular coronario. Nuestro objetivo fue comparar parámetros angiográficos de flujo coronario en pacientes con infarto de miocardio con supradesnivel del ST que habían consumido cocaína versus pacientes que no habían consumido.

Métodos: Estudio analítico observacional con selección aleatoria de los controles. Se midió el recuento de cuadros TIMI y el "blush" Miocárdico.

Resultados: De un total de 1294 pacientes cuyo infarto se trató con angioplastía primaria, 59 declararon haber consumido cocaína previo al evento. Los controles fueron 142 pacientes sin consumo de cocaína previo, seleccionados aleatoriamente. Los consumidores eran más jóvenes ( $38 \pm 10$ vs $58 \pm 10$ años, $\mathrm{p}<0,001)$, con predominio de hombres $(89.8 \%$ vs $77.3 \%, \mathrm{p}=0,039)$, menor proporción de hipertensión arterial $(8.5 \%$ vs
$58 \%, \mathrm{p}<0,001)$, y de Diabetes Mellitus tipo 2 (6,8\% vs $26,1 \% \mathrm{p}=0,002)$. Tenían menos arterias con estenosis $>70 \%$ (4,9 versus $40,7 \%, \mathrm{p}<0,001)$ y menor recuento de cuadros TIMI inicial $(61 \pm 35$ vs $84 \pm 28$ cuadros, $\mathrm{p}<0,001)$. El implante de stents fue menos frecuente en los consumidores ( $37,3 \%$ vs $89,9 \%, \mathrm{p}<0.001)$. Al término de la angioplastía, los consumidores tuvieron peor recuento de cuadros que los controles $(37 \pm 222$ versus $29 \pm 17$ cuadros, $p=0,003$ ). Sólo la variable "consumidores" fue significativa para peor cTFC (OR 2,6, $\mathrm{p}=0,049$, $95 \%$, IC 1,003-6,721). El "blush" miocárdico no fue diferente entre los grupos.

Conclusiones: El infarto asociado a consumo de cocaína se presenta en hombres que tienen menos factores de riesgo tradicionales, y menos prevalencia de estenosis coronarias significativas. Los consumidores presentan mayor deterioro del flujo coronario microvascular post angioplastía.

\section{Correspondencia:}

Dr. Eduardo Guarda Salazar

División de Enfermedades Cardiovasculares

Facultad de Medicina, Pontificia Universidad Católica de Chile

Eguarda@med.puc.cl 


\section{Microvascular coronary blood flow deteriorates after percutáneous coronary angioplasty in cocaine consumers}

Introduction and objective: Cocaine consumption may induce microvascular coronary blood flow deterioration. Our objective was to compare microvascular coronary blood flow in patients with acute myocardial infarction and ST elevation, with or without prior consumption of cocaine.

Methods: This was a case - control (1:2) observational analytic study. We measured TIMI frame count and Myocardial Blush Grade in all patients.

Results: From a total of 1294 primary angioplasty, 59 patients declared cocaine consumption prior to the event. Controls were 142 patients. Consumers were younger ( $38 \pm 10$ vs $58 \pm 10$ years, $\mathrm{p}<0.001)$, predominantly male ( $89.8 \%$ vs $77.3 \%, \mathrm{p}=0.039)$, had less arterial hypertension $(8.5 \%$ vs $58 \%, \mathrm{p}<0.001)$ and type 2 Diabetes Mellitus (6.8\% vs $26.1 \%, \mathrm{p}=0.002$ ). Stenosis $>70 \%$ were less frequent in consumers than in controls $(4.9 \%$ vs $40.7 \%, \mathrm{p}<0.001)$. They had lower baseline TIMI frame count $(61 \pm 35$ vs $84 \pm$ 28 frames, $\mathrm{p}<0.001)$ and stent implantation was less common $(37.3 \%$ vs $89.9 \%$, p $<0.001)$. Final TIMI frame count was $37 \pm 22$ in consumers vs $29 \pm 17$ frames in controls, $p=0.003$ ). Cocaine was a statistically significant predictor of poor TIMI frame count in a multivariate analysis (OR 2.6, $\mathrm{p}=0.049,95 \% \mathrm{CI}$ 1.003-6.721). Myocardial Blush was not different between groups.

Conclusions: Cocaine consumption associated to STEMI is more frequent in young male patients, with fewer cardiovascular risk factors and less severe angiographic lesions. Cocaine consumers had higher prevalence of decreased coronary microvascular flow after primary angioplasty.

Key words: Acute myocardial infarction with ST elevation, Percutaneous Coronary Intervention, Cocaine/adverse effects. 


\section{Introducción:}

La cocaína es una de las drogas ilícitas recreacionales más usadas a nivel mundial ${ }^{1}$. La prevalencia en el consumo de cocaína en Chile alcanzó el $0,7 \%$ de la población entre 2006 y $2010^{2}$. El consumo de cocaína se ha asociado a infarto del miocardio (IAM), insuficiencia cardíaca, hemorragia cerebral y otras afecciones cardiovasculares ${ }^{3}$.

El IAM asociado al consumo de cocaína se presenta más frecuentemente en pacientes jóvenes, de sexo masculino y con una muy baja prevalencia de factores de riesgo ${ }^{4}$. Los elementos que se han destacado en su etiopatogenia son vasoespasmo y alta carga trombótica ${ }^{5}$.

Existe escasa información respecto a las características de la microcirculación en los pacientes con IAM asociado a consumo de cocaína tratados mediante angioplastía primaria (AP). Considerando que los mecanismos de acción de la cocaína pueden tener efecto sobre la microcirculación, los pacientes consumidores sometidos a AP por IAM c/SDST podrían presentar mayor deterioro del flujo micro-vascular en comparación con pacientes no consumidores.

El objetivo de este trabajo fue comparar parámetros angiográficos de microcirculación en pacientes con IAM c/ SDST que habían consumido versus pacientes que no habían consumido cocaína. Para ello se evaluó la microcirculación de forma cuantitativa a través de conteo de cuadros TIMI (cTFC) y cualitativa mediante "blush" Miocárdico $(\mathrm{BM})^{6}$.

\section{Métodos}

Se realizó un estudio analítico observacional caso:control ${ }^{1-2}$ para evaluar las diferencias cuantitativas y cualitativas de la microcirculación coronaria post AP entre pacientes con IAM c/SDST que habían consumido versus pacientes que no habían consumido cocaína. Para ello, se efectuó una revisión sistemática de los registros clínicos y angiográficos de la base de datos del total de pacientes sometidos a AP por IAM c/SDST en el laboratorio de hemodinamia del Hospital Doctor Sótero del Río, desde Enero del 2009 hasta Abril del 2013. El protocolo fue aprobado por el Comité de ética institucional.

\section{Pacientes:}

Se catalogó como "casos" a los 59 pacientes con IAM c/ SDST de menos de 12 horas de evolución tratados con AP y que declararon haber consumido cocaína en relación al cuadro actual, y no tenían criterios de exclusión. Para el grupo control se realizó un selección aleatoria del total de pacientes sometidos a AP, que negaron consumo de cocaína. Fueron excluidos los pacientes en shock cardiogénico, los que recibieron trombolisis IV y, en el grupo control, los que refirieron haber consumido alguna vez cocaína. No hubo exclusión por género ni edad. Para el calculó del tamaño muestral se consideró el número necesario de controles en base a la proporción hipotética de $30 \%$ de pacientes con cuenta de cuadros TIMI (cTFC) menor o igual a 23, cifra que se considera como buen resultado microcirculatorio $^{7}$. Así, se estableció que para $\mathrm{p}=0.05 \mathrm{y}$ poder estadístico de $80 \%$, para los 59 casos se requerían 142 controles.

\section{Angioplastía primaria}

Los pacientes recibieron aspirina, nitroglicerina IV, y heparina no fraccionada, en el servicio de urgencia. La coronariografía y la angioplastía fueron realizadas de acuerdo a protocolos estándar en nuestro país incluyendo carga de heparina no fraccionada para mantener un tiempo de coagulación activado > $300 \mathrm{seg}$ y administración de $600 \mathrm{mg}$ de clopidogrel ${ }^{8}$. El uso de aspiración manual de trombos, inhibidores IIb/IIIa, nitroglicerina IV, adenosina, verapamilo o nitroprusiato intracoronario (IC), y predilatación o postdilatación con balón, quedaron a discreción del operador.

\section{Mediciones angiográficas}

Dos observadores ciegos a la información de consumo de cocaína realizaron evaluación cuantitativa del flujo microvascular a través de cTFC pre y post angioplastía, y cualitativa con BM luego de terminada la angioplastía.

Las proyecciones para realizar el cFTC y BM se realizaron de acuerdo a Hamada $\mathrm{S}$ et $\mathrm{al}^{7}$. Brevemente, se realizaron 2 filmaciones en proyecciones ortogonales, de 15 segundos de duración, pre y post angioplastía, la última sin alambre guía. Los formatos angiográficos fueron de 22 pulgadas. Las mediciones angiográficas cuantitativas fueron efectuadas en fin de diástole. Se consideraron como significativas estenosis $>$ de $70 \%$.

\section{Análisis estadístico:}

Las características basales de los pacientes (edad, sexo, comorbilidades, localización del infarto, cTFC inicial) se expresan como promedios \pm DS y porcentajes.

La comparación del cTFC entre los resultados basales y post angioplastía entre los grupos se realizó con t de student para muestras independientes o la prueba U Mann Whitney de no cumplir con los criterios de aplicación de pruebas paramétricas.

El análisis comparativo del grado de BM basal y post angioplastía entre los grupos se hizo mediante la prueba de 
$\mathrm{X}^{2}$ para muestras independientes para variable de agrupación con $\mathrm{K} \geq 3$ grupos. Para comparar entre ambos grupos el porcentaje de casos que obtuvieron $\mathrm{cTFC} \leq 23$ post angioplastia, se utilizó $\mathrm{X}^{2}$ para muestras independientes. Se realizó análisis uni y multivariado para el cálculo de Odds Ratio (OR) para "mal resultado angiográfico microvascular" (definido como cTFC final $>23$ cuadros) ${ }^{7-9}$.

\section{Resultados}

Entre Enero del 2009 y Abril del 2013 se realizaron 1294 estudios coronarios de urgencia por IAM c/SDST, entre los cuales hubo 59 pacientes que refirieron en forma explícita haber consumido cocaína en las horas previas al inicio de los síntomas del IAM cocaína (Grupo Cocaína, GCoca); corresponden al 4,5\% del total. El grupo control (GC) quedó constituido por 142 pacientes.

Características clínicas basales: El GCoca fue en promedio 20 años menor que los controles $(38 \pm 10$ años versus $58 \pm 10$ años, $\mathrm{p}<0,001)$, tenía mayor porcentaje de hombres $(89,8 \%$ versus $77,3 \%, p=0,039)$, menor presencia de HTA $(8,5 \%$ versus $58 \%, \mathrm{p}<0,001)$, de diabetes tipo $2(6,8 \%$ versus $26 \%, \mathrm{p}<0,002)$ y de angioplastías previas $(1,7 \%$ versus $9,9 \%, \mathrm{p}=0,045)$. No hubo diferencias en dislipidemia, tabaquismo, obesidad o IAM antiguo (Tabla 1).

\begin{tabular}{|c|c|c|c|}
\hline \multicolumn{4}{|c|}{ IAM con SDST } \\
\hline & Grupo Coca & Grupo Control & $p$ \\
\hline Número pacientes & 59 & 142 & \\
\hline Edad (años $\pm D E)$ & $38 \pm 10$ & $57 \pm 10$ & $P<0.001$ \\
\hline Hombres (\%) & 89 & 77 & $P<0.03$ \\
\hline Hipertensión Arterial (\%) & 8.5 & 58 & $P<0.001$ \\
\hline Diabetes Mellitus tipo $2(\%)$ & 6.8 & 26 & $P<0.002$ \\
\hline Dislipidemia (\%) & 13.6 & 11.3 & NS \\
\hline Tabaquismo (\%) & 52.5 & 53.5 & NS \\
\hline Obesidad (\%) & 5.1 & 7 & NS \\
\hline Angioplastía previa (\%) & 1.7 & 9.9 & $P<0.045$ \\
\hline
\end{tabular}

Angiografía y procedimientos efectuados: El GCoca tenía mayor porcentaje de pacientes con arterias coronarias sin estenosis angiográficas significativas ( $>70 \%$ estenosis) versus el GC (40,7\% versus $4,9 \%, \mathrm{p}<0.001)$, menor cTFC inicial $(61 \pm 35$ versus $84 \pm 28$ cuadros, $p<0,001)$. No se evidenciaron diferencias en relación a la arteria comprometida .

En comparación con el GC, en el GCoca se realizó un menor porcentaje de aspiración de trombos $(20,3 \%$ versus $43 \%, \mathrm{p}=0,002)$, predilatación $(27,1 \%$ versus $56,3 \%$, $\mathrm{p}<0.001)$, implante de Stents $(37,3 \%$ versus $89,9 \%$, $\mathrm{p}<0,001)$ y postdilatación $(6,8 \%$ versus $26,1 \%, \mathrm{p}=0,002)$, (Tabla 2)

Tabla 2: Características de la angioplastía en consumidores de cocaína y sujetos controles

\begin{tabular}{|l|r|r|r|}
\hline & Grupo Coca & Grupo Control & $p$ \\
\hline Aspiración de trombos (\%) & 20.3 & 43 & 0.002 \\
\hline Medicamentos intracoronarios (\%) & 1.7 & 0 & NS \\
\hline Estreptoquinasa intracoronaria (\%) & 3.4 & 0 & NS \\
\hline Implante Stent & 37.3 & 89.9 & $p<0.001$ \\
\hline Predilatación (\%) & 27.1 & 56.3 & $\mathrm{P}<0.001$ \\
\hline Postdilatación (\%) & 6.8 & 26.1 & $\mathrm{p}=0.002$ \\
\hline Otro vaso tratado el mismo día (\%) & 3.4 & 4.2 & $\mathrm{NS}$ \\
\hline
\end{tabular}

Medicamentos intracoronarios: adenosina, verapamilo, nitroprusiato

\section{Evaluación de la microcirculación posterior a la angio-} plastía:

Después de la AP, no encontramos diferencias significativas en cuanto al BM $(2,46 \pm 0,7$ en el GCoca versus 2,35 $\pm 0,7$ en el GC, $p=0,621$ ).

Respecto del cTFC, los pacientes del GCoca presentaron un significativo deterioro del flujo microvascular $(37 \pm 22$ cuadros) en comparación con el GC ( $29 \pm 17$ cuadros), $\mathrm{p}=0,003)$.

El flujo microvascular post AP, definido como un cTFC $>23$ cuadros, estaba presente en el 42,3\% del GCoca y en sólo $21.1 \%$ del GC $(\mathrm{p}=0,005)$.

Al comparar pacientes de ambos grupos en quienes se implantó un Stent, evidenciamos que el cTFC final fue peor en el GCoca ( $38 \pm 18$ versus $29 \pm 15$ cuadros, $\mathrm{p}=0,003)$ (Tabla 3).

\begin{tabular}{|c|c|c|c|}
\hline \multicolumn{4}{|c|}{$\begin{array}{c}\text { Tabla 3: Evaluación de la microcirculación coronaria en } \\
\text { consumidores de cocaína y sujetos controles sometidos } \\
\text { angioplastía primaria }\end{array}$} \\
\hline & Grupo Coca & Grupo Control & Significancia \\
\hline cTFC Basal & $61 \pm 35$ & $84 \pm 28$ & $p<0.001$ \\
\hline cTFC Post angioplastía & $37 \pm 22$ & $29 \pm 17$ & $P<0.003$ \\
\hline cTFC < $23(\%)$ & 21.1 & 42.3 & $P<0.005$ \\
\hline "blush" Miocárdico & $2.46 \pm 0.7$ & $2.35 \pm 0.7$ & NS \\
\hline
\end{tabular}

TIMI $=$ Thrombolysis in Myocardial Infarction

$c T F C=$ Conteo de cuadros TIMI

Análisis Multivariado para la predicción de cTFC sobre 23 cuadros: El análisis univariable para evaluar el resultado cTFC $>23$ cuadros (pobre resultado angiográfico) mostró las siguientes variables estadísticamente significativas: consumo de cocaína, DM2, HTA, lesiones angiográficas significativas, edad y sexo. Sólo la variable consumo de cocaína, ajustada por el resto de las variables, 
fue estadísticamente significativa para cTFC $>23$ cuadros, con un OR de 2,6 (IC 95\% 1,003-6,721, p=0,049).

\section{Discusión}

Nuestro estudio confirma que el IAM c/SDST asociado a consumo de cocaína tiene diferencias importantes con el IAM c/SDST de los no consumidores. Similarmente a lo observado en otros estudios ${ }^{4}$, estos sujetos son más jóvenes, con menos factores de riesgo cardiovascular y con un mayor porcentaje de estenosis coronarias no significativas. En comparación con el GC, los pacientes del GCoca presentaron mayor alteración del flujo coronario microvascular medido por cTFC. Aunque el cTFC inicial del GC era menor que el GCoca, evidenció una mejoría significativa al finalizar la intervención, lo que sugiere que los pacientes del GCoca pueden tener mayor disfunción endotelial expresada a través del deterioro de la microcirculación que provoca la angioplastía. Este patrón de alteración de la microcirculación estuvo presente en los pacientes consumidores, independiente de si existían, o no, lesiones coronarias significativas, ni de la implantación, o no, de Stents. Entre los diversos métodos para evaluar el flujo microvascular, utilizamos el cTFC, que sirve para evaluar de forma cuantitativa el flujo epicárdico y la microcirculación. Éste ha demostrado ser un buen marcador de eventos clínicos a largo plazo, estableciendo como buen pronóstico el tener $\leq$ a 23 cTFC, además de ser reproducible, con baja variabilidad inter e intra observador $(1-2 \%)^{7}$. Los consumidores de cocaía tenían un peor cTFC compardo con los controles, lo que sugiere la posibilidad de peor pronóstico en estos pacientes. Por otra parte, no encontramos diferencias significativas en el BM entre los 2 grupos. Esto puede deberse a que se trata de una medición cualitativa, con una distribución anormal, lo que resta poder estadístico opara detectar una posible diferencia de BM entre consumidores y controles.

Como mostramos en el análisis multivariado, sólo la variable consumo de cocaína, ajustada por el resto de las variables, fue estadísticamente significativa (OR de 2,6) para cTFC sobre 23 cuadros, lo que es considerado como un mal resultado angiografíco sugerente de peor pronóstico a futuro ${ }^{7}$.

En pacientes con IAM c/SDST tratados con trombolisis, Weber y cols ${ }^{10}$ encontraron que el flujo de la microcirculación fue mejor en los consumidores de cocaína que en los no consumidores. Esta diferencia respecto de nuestros hallazgos puede sugerir que en los pacientes consumidores tratados con AP y stents coronarios se requieran medidas antitrombóticas adicionales.

Nuestro estudio tiene limitaciones. Se trata de un estudio observacional y el antecedente anamnéstico de consumo de cocaína no se había corroborado con exámenes de laboratorio previo al IAM.

En conclusión, el consumo de cocaína en IAM c/SDST se asocia a pacientes más jóvenes, con menor número de factores de riesgo cardiovascular y menor magnitud de lesiones coronarias significativas en comparación a población no expuesta a su consumo. Después de la AP, la microcirculación en los consumidores está más comprometida que en los no consumidores.

Agradecimientos: A Karla Zuanic, María Mestas y a Úrsula Valdivia por el cuidado de los pacientes y por el acucioso registro de los datos clínicos. 


\section{Referencias:}

1. World Drug Report 2012. United Nations office for Drugs and Crime. Disponible en: http//www.unodc.org/unodc/en/dataandanalysis/WDR-2012.html.

2. Senda. Gobierno de Chile. Noveno estudio nacional de drogas en población general en Chile. Disponible en http://www.senda.gob.cl

3. SCHWARTZ BG, REZKALLA S, KLONER RA. Cardiovascular effects of cocaine. Circulation. 2010; 122: 2558-69.

4. BOSCH X, LOMA-OSORIO P, GUASCH E, NOGUÉ S, ORTIZ JT, SÁNCHEZ M. Prevalencia, características clínicas y riesgo de infarto de miocardio en pacientes con dolor torácico y consumo de cocaína. Rev Esp Cardiol 2010; 63: 1028-34

5. MARAJ S, FIGUEREDO VM, LYNN MORRIS D. Cocaine and the heart. Clin Cardiol. 2010; 33: 264-9

6. GIBSON CM, CANNON CP, DALEY WL, DODGE JT JR, ALEXANDER B JR, MARBLE SJ, et al. TIMI frame count: a quantitative method of assessing coronary artery flow. Circulation. 1996; 93: $879-88$
7. HAMADA S, NISHIUE T, NAKAMURA S, SUGIURA T, KAMIHATA H, MIYOSHI H, et al. TIMI frame count immediately after primary coronary angioplasty as a predictor of functional recovery in patients with TIMI 3 reperfused acute myocardial infarction. J Am Coll Cardiol. 2001; 38: 666 - 71

8. GUARDA E, PRIETO JC, SANHUEZA P, DAUVERGNE C, ASENJO R, CORBALÁN R. Guías 2009 de la Sociedad Chilena de Cardiología para el tratamiento del Infarto Agudo del Miocardio con supradesnivel del ST. Rev Chil Cardiol 2009; $28,223-254$

9. LINDEFJELD DS, GUARDA E, MÉNDEZ M, MARTÍNEZ A, PÉREZ O, FAJURI A, et al. Microvascular coronary flow comparison in acute myocardial infarction angioplasty treated with a mesh covered stent (MGUARD stent) versus bare metal stent: MICAMI-MGUARD. Cardiovasc Revasc Med. 2013; 14: $4-8$

10. WEBER JE, HOLLANDER JE, MURPHY SA, BRAUNWALD E, GIBSON CM. Quantitative comparison of coronary artery flow and myocardial perfusion in patients with acute myocardial infarction in the presence and absence of recent cocaine use. J Thromb Thrombolysis 2002;14:239-45. 